\title{
LA ETNOBIOLOGÍA DESDE LA PERSPECTIVA LINGÜÍSTICA DE LOS SOLEGA
}

\author{
Juan Sebastian Orjuela Forero \\ Universidad del Tolima
}

Si, Aung (2016). The Traditional Ecological Knowledge of the Solega: A Linguistic Perspective. Springer. DOI 10.1007/978-3-319-24681-9

La etnobiología, es una rama de la biología que se centra en el estudio de los conocimientos tradicionales, partiendo del manejo tradicional (o conocimiento ecológico tradicional, como lo menciona el autor) de las comunidades ancestrales, ya que estos conocimientos son de gran importancia en el avance de ciencias como la biología en la actualidad; sin embargo, estos conocimientos muchas veces son vistos desde unas perspectiva biológica, y no vinculan el contexto lingüístico, social y psicológico que atraviesan las comunidades en donde se almacena la información que se transmite a través de la tradición oral en muchos casos.

Aung Si, autor del libro The Traditional Ecological Knowledge of the Solega: A Linguistic Perspective, documenta los conocimientos tradicionales de los Solega, grupo étnico ubicado en las colinas de Biligiri Rangaswamy, al suroeste de Karnataka (India), incorporando sus dobles títulos de doctorado en neurociencia y lingüística.

La financiación de este manuscrito, como lo expresa el autor, fue gracias a la Universidad Nacional Australiana y el Hans Rausing Endangered Languages Project, publicado bajo el sello editorial Springer en el año 2016. El libro presenta un atractivo desde el punto de vista académico, abarcando el concepto de la etnobiología de una manera accesible para la comunidad en general; se divide en ocho capítulos, en los cuales aborda: la importancia de la lingüística en las investigaciones sobre etnobiología, la etnoclasificación, la importancia y clasificación de las plantas en la comunidad, la etnoornitologia, 
el concepto de paisaje desde la perspectiva de los Solega, un análisis semiótico de la comunidad, y los conocimientos sobre las abejas de una comunidad no apícola; en el capítulo final realiza una discusión sobre la naturaleza de la investigación centrada en el conocimiento ecológico tradicional.

El primer capítulo realiza una presentación de la comunidad Solega y las preguntas centrales para la investigación. Aborda de manera elegante y precisa el concepto de la etnobiología, documentando los "conceptos situacionales y socioculturales" que considera necesarios para la comprensión de la situación de la comunidad Solega, creando apartados para que los lectores comprendamos la organización territorial, la gobernanza, las creencias religiosas, la situación socioeconómica y nutricional, la percepción de la población hacia su entorno, y el impacto que ha tenido la modernización en la cultura, lengua y transmisión de los conocimientos tradicionales en la comunidad. El autor hace un esfuerzo para presentar los hallazgos obtenidos como un cuerpo de conocimiento, y no simplemente como aspectos lingüísticos en la etnobiología centrados en la clasificación taxonómica.

El cierre del capítulo evidencia el arraigo de los Solega hacia el bosque que los rodea, amenazado brutalmente por la presencia de especies invasoras que desplazan tanto a la fauna como la flora autóctona.

En esta línea argumental, en los capítulos 2 al 4, el autor aborda el tema de la etnoclasificación (haciendo un apartado especial para plantas y aves en los capítulos 3 y 4 , respectivamente), desde una perspectiva cultural y funcional (con respecto a las utilidades que tanto plantas como animales pueden tener para la comunidad), contrastando esta visión con los supuestos "universales" que expresa Berlín (1992) en su monografía Ethnobiological classification. Aung Si, de manera concreta, contraargumenta los supuestos de Berlín basándose no solo en la percepción obtenida de la clasificación cultural realizada por los Solega (en la que se evidencia que la familia Orchidaceae, al no poseer una utilidad para la comunidad, son simplemente descritas como "baccanike", a pesar de su diversidad morfológica observable, por ejemplo), sino también soportada por diversos estudios publicados por Baker o Stross (por mencionar solo algunos de los ejemplos expuestos en el capítulo). El autor menciona que los estudios etnobiológicos deben incluir información relacionada sobre la utilización de los términos en la comunicación.

En el siguiente capítulo, se presentan 27 extractos de entrevistas en las cuales evidencia la variedad en la terminología que utilizan los Solega para 
referirse a su entorno (identificando y distinguiendo entre cerca de 14 hábitats, cada uno con un nombre y características específicas), así como la conciencia de sus habitantes con respecto a la estacionalidad en la región. De una manera muy fluida e interesante, el autor nos presenta, además de la información relacionada con la hidrología y paisaje, fotografías y dibujos esquemáticos que ayudan a los lectores en una inmersión más real en el mundo de los Solega. Otro factor importante para la vida de los Solega, son los signos tanto implícitos como explícitos que están a su alrededor; ellos mejor que nadie han aprendido a conocer y a aprender de las señales que les brinda su entorno para comprender las relaciones simbióticas y antagónicas que existen entre los diversos seres que conviven en la zona, y extrapolarlas a lo que los seres humanos viven bajo las mismas presiones meteorológicas, como se explica en el capítulo 6.

Bajo esta perspectiva, en el séptimo apartado se abordan los conocimientos de los Solega sobre las abejas (especie estudiada con anterioridad por el autor en el desarrollo de su doctorado en neurociencia), usando los conocimientos y prácticas desarrolladas como agricultores, y cazadores/recolectores para contraargumentar los "universales" propuestos por Berlín, como lo ha hecho a lo largo de los demás capítulos presentados durante todo el transcurso del libro. De este modo, se detallan las prácticas apícolas de los Solega por medio de transcripciones de los relatos y canciones que utilizan los recolectores durante el proceso de obtención de miel, así como el relato textual de uno de sus colaboradores, detallando con una gran precisión la ubicación de los árboles de los cuales se realiza la obtención de la miel. El desarrollo de este capítulo en especial, muestra de una manera fluida la comprensión de los Solega del comportamiento, patrones de migración, reproducción y ecología de las abejas, basados solo en observaciones limitadas por las épocas en las que tienen acceso a las colmenas.

El capítulo de cierre, presenta de una manera más compacta la discusión que ha venido presentando durante el desarrollo de cada uno de los capítulos previos, remarcando que es necesario el análisis de un solo idioma, para poder refutar con fuerza cada uno de los "universales" tratados por Berlín (1992).

En general, la valoración que me llevo del libro es muy favorable, gracias a la manera sencilla de abordar temas tan complejos como lo pueden ser los sistemas de clasificación basados en conocimientos ecológicos tradicionales, volviendo estos conocimientos accesibles para cualquier persona interesada en el tema, independientemente del campo de estudio. Refuerza de manera 
clara y objetiva la importancia de que los estudios etnobiológicos se complementen con estudios lingüísticos para enriquecer los resultados desde el punto de vista de la comunidad de estudio, y sienta las bases para que lingüistas interesados en el estudio de conocimientos tradicionales de comunidades no malinterpreten los conceptos clave de la literatura biológica y etnobiológica.

Finalmente, este libro es crucial para tomarlo como ejemplo de cómo elaborar de manera objetiva y muy bien desarrollada, los estudios de documentación de conocimientos tradicionales con comunidades ancestrales. La presentación de la metodología de investigación es muy transparente y reflexiona sobre los aspectos que se pueden trabajar para mejorar el resultado final. Este volumen, es una de las mejores documentaciones que he observado de los conocimientos ecológicos tradicionales desde la perspectiva lingüística de la comunidad, y es un gran ejemplo para algo que espero, sea tendencia en las investigaciones posteriores centradas en registrar, preservar y compartir los conocimientos tradicionales en todo el mundo.

Juan Sebastian Orjuela

jsebastianorjuela@ut.edu.co 